\title{
The Application of Scattered Light in the Metrology of Planar Waveguides
}

\author{
K. GuT* \\ Department of Optoelectronics, Silesian University of Technology \\ Akademicka 2A, 44-100 Gliwice, Poland
}

The paper presents a measuring stand which permits to determine the attenuation and modal birefringence of planar waveguide structures. The light is introduced into the waveguide through a prismatic coupler. This permits to determine attenuations of the subsequent modes. Mounting the polarizer in front of the lens of the camera it becomes possible to determine on the same test stand the modal birefringence of orthogonal pair of modes of the same order.

PACS: 42.25.Ja, 42.25.Lc, 42.25.Fx

\section{Introduction}

Optical waveguide structures constructed in compliance with the technique of planar optics, applied also in telecommunication (among others as modulators, multiand demultiplexer) are thanks to many advantages, unattainable in the technology of optical fiber, more and more widely used in the production of sensor systems monitoring physical quantities, biological and chemical processes, as well as numerous parameters in various domains of science and industry. This is proved by the constantly growing number of scientific publication presenting new solution and improvements of optical sensors operating on the principle of contacting the optic wave with the monitored medium.

One of the most important parameters characterizing the obtained waveguide of integrated optics is, besides the optical properties, the attenuation defined as the loss of optic power per unit path of propagation in the case of systems of integrated optics; the proper choice of the method of measuring the attenuation is much more complicated than in the case of optical fiber, chiefly due to is different geometry and structure, its appropriation and the difference range of the investigated losses [1-9]. Therefore, it is impossible to develop one universal method of measuring the attenuation, and most of the methods applied so far are mostly designs assigned for measurements carried out in laboratories.

The fundamental principle of the measurement of losses due to propagation comprises the introduction into one end of the waveguide of a certain quantity of optical power and measurement of the power output at the other end [1]. Such a simple approach involves, however, several inherent problems and some inaccuracy of those measurements due, among others, to the fact that the amount of losses at the input and the output is unknown, whose losses may distort considerably the actual value of propagation losses, whereas in the case of investigations concerning multimode waveguides the losses of the individual mode cannot be assessed separately.

\footnotetext{
* e-mail: kgut@polsl.pl
}

Figure 1 illustrates the general division of measurement methods of the attenuation of planar waveguides. Generally, two fundamental techniques of measurements are to be distinguished, differing from each other mainly by the way of letting out the output light from the waveguide: transmission method - basing on measurements light power at the output as a function of the distance of propagation, and by scanning the scattered light from the waveguide as a function of the distance. Complementing the technique of measurements of attenuation is another group of methods, each of them basing on the application of different physical phenomenon, e.g. the photothermal effect of the deflection of the beam, interference or fluorescence.

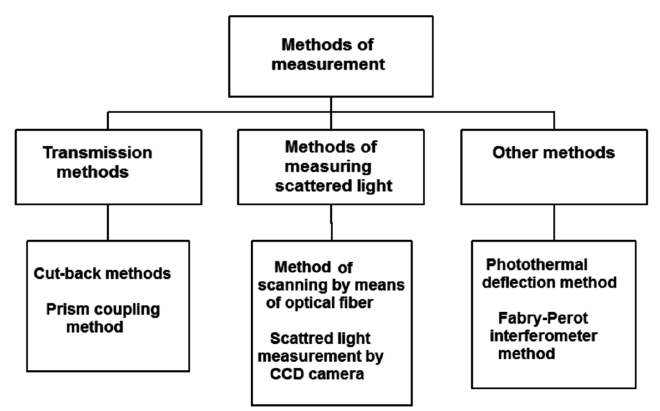

Fig. 1. General specification of the methods of measuring the attenuation of waveguides integrated optics.

Another important parameter characterizing planar waveguides is the modal birefringence [10-13]. Orthogonal $m$-order modes propagating in a planar waveguide differ from each other because of the propagation constants $\beta_{\mathrm{TE} m} \neq \beta_{\mathrm{TM} m}$. Because of this, they propagate with different phase velocities. That is why after passing a path which is $x$-long between the propagating modes $\mathrm{TE}_{m}$ and $\mathrm{TM}_{m}$ the phase difference is $\Delta \Phi$, defined as [10]:

$$
\Delta \Phi=\left|\beta_{\mathrm{TE}_{m}}-\beta_{\mathrm{TM}_{m}}\right| x,
$$

where $\Delta \Phi$ - phase difference between the propagating modes, $\beta_{\mathrm{TE} m}, \beta_{\mathrm{TM} m}-\mathrm{TE}$ and TM $m$-order mode propagation constants. 
If the phase difference between the modes amounts to $2 \pi$ after passing the path of propagation $L_{\mathrm{B} m}$, it is called the beat length $m$-pair of orthogonal modes [10]:

As

$$
2 \pi=\left|\beta_{\mathrm{TE}_{m}}-\beta_{\mathrm{TM}_{m}}\right| L_{\mathrm{B}_{\mathrm{m}}} .
$$

$$
\beta=\frac{2 \pi}{\lambda} N_{\mathrm{eff}}
$$

we get

$$
\left|N_{\mathrm{eff}_{\mathrm{TE}}}-N_{\mathrm{eff}_{\mathrm{TM}}}\right|=\frac{\lambda}{L_{\mathrm{B}_{\mathrm{m}}}},
$$

where $N_{\text {effTE }}\left(N_{\text {effTM }}\right)$ - effective refractive index mode TE(TM), $\lambda$ - wavelength, $N_{\text {effTE }}-N_{\text {effTM }}-$ modal birefringence.

The functioning of most waveguide planar sensors is based on the phenomena of differential interference and detected by measuring the phase difference $\Delta \Phi$ between the propagating modes $\mathrm{TE}_{m}$ and $\mathrm{TM}_{m}$ at the end of the waveguide [14-26].

Several methods of measuring the modal birefringence of planar waveguide structures have been developed. The effective refractive indices of modes are determined by means of a prism coupler and then also their differences [25]. Another method is based on an analysis of the polarization states of the output light in response to known input polarization states [26]. When superluminescent diodes (SLD) are applied, the spectral interference method permits to determine the modal birefringence [27]. For this purpose an immersion coupler may be used which allows the light to leave from the two waveguide modes continuously and to determine the beat length [10]. If only one mode is excited and a spot excitation of displacement is applied, coupling some part of energy with the orthogonal mode also this value can be determined [28].

\section{Determination of the attenuation}

Measurements of attenuation applying the method of recording the intensity of scattered light are absolutely reliable, non-destructive, and fast in their execution. Concerning this method it is assumed that the intensity of scattered light is proportional to the intensity of light in the waveguide. Such an assumption permits to determine the attenuation of the waveguide, and thus also to estimate how much of the entire power rating introduced into the waveguide gets lost due to absorption and dissipation. Scattered light is to be observed on the upper surface of the waveguide as visible radiation, its direction coinciding with the direction of the light introduced into the waveguide. The dependence of intensity of the scattered light along the path of propagation is expressed by the following relation [7]:

$$
I_{s}=I_{0} \mathrm{e}^{-\alpha x},
$$

where $x$ - path of propagation, $I_{0}$ - power rating introduced into the waveguide at the point $x=0, \alpha$ - linear absorption coefficient $[1 / \mathrm{cm}]$.

By means of the relation (1) the attenuation coefficient can be determined as the directivity index of the straight line of the linear matching function $I_{s}=f(x)$.
Practically, the value of the attenuation coefficient $\alpha$ is expressed in the logarithmic scale, i.e. in $\mathrm{dB} / \mathrm{cm}$. This can be achieved by a conversion of the scale [9]:

$$
\ln I_{s}=\ln I_{0}-\alpha x \quad \Leftrightarrow \quad 10 \log I_{s}=b-a_{\mathrm{dB}} x .
$$

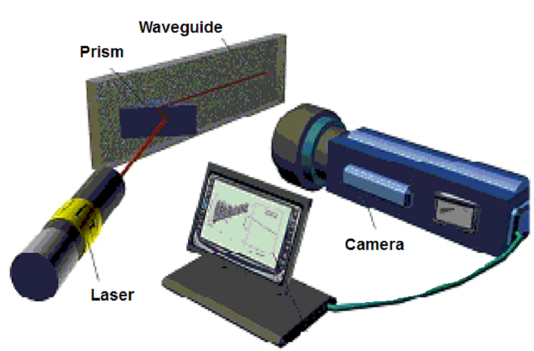

Fig. 2. Experimental setup.

The intensity of scattered light was recorded by means of a CCD camera, provided with an optical system, which permits to focus on the surface of the waveguide. The obtained images were analyzed in compliance with the relation (2), and the resulting value of the attenuation coefficient $\alpha_{\mathrm{dB}}$, was assessed to be the value of the coefficient of the straight line matching the function $10 \log I_{s}=f(x)$.

This test stand is shown in Fig. 2. It is equipped with a grip holding the waveguide, a CCD camera, and a computer with a specially assigned software. The holder of the waveguide is provided with a manipulator which permits to adjust the position of the structure in relation to the camera. In the arm of the holder there is a laser as well as a diaphragm. The CCD camera is mounted above the upper plane of the waveguide. It possesses an optical system with an adjustable focal distance. It is of extreme importance that the camera is equipped with an adequate CCD transducer with linear sensitivity characteristics in the given range of wavelength in which the investigations are being run [10].

For the purpose of introducing light into the waveguide a prism coupler was applied. The choice of proper polarization was accomplished by the proper rotation of the polarizer. The image was detected by means of a black-and-white camera GKB (CB23803SA 1/3" IR BW CCD). The planar single mode structure of the waveguide was made in a glass substrate (soda-lime) applying the ion-exchange technique $\mathrm{K}^{+}-\mathrm{Na}^{+}$. Figure 3 shows the recorded photo and linear fit to the logarithm of the intensity of the scattered light as a function of the light propagation path of light in the waveguide.

The measurements were carried out for the wavelength of light of $635 \mathrm{~nm}$. The attenuation of the mode presented in Fig. 3 amounts to $1.8 \mathrm{~dB} / \mathrm{cm}$.

\section{Determination of the beat length}

Scattered light can be used to determine the modal birefringence.x By adjusting the polarization input beam appropriately (on the test stand presented in Fig. 2), it was possible to excite the modes $\mathrm{TE}_{i}$ and $\mathrm{TM}_{i}$ simultaneously. A definite pair of modes was selected by changing 


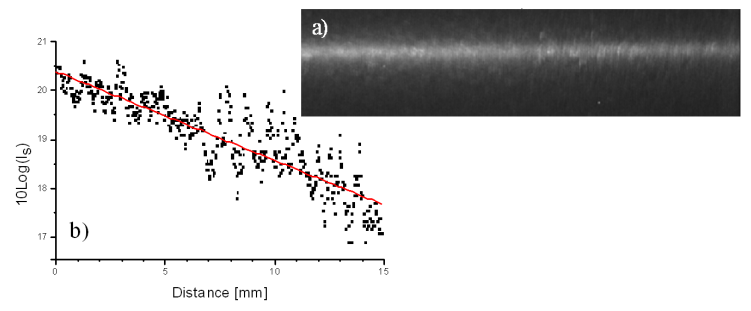

Fig. 3. (a) Image captured by the CCD camera. (b) Logarithmic dependence of the intensity of scattered light. Analyzed images of the excited mode ( $\mathrm{TE}_{0}$ mode).

the angle of the beam input to the prism. Placing an additional polarizer in front of the camera we can observe oscillations of the intensity of light (in the direction of its propagation) connected with the interference of scattered light from the modes TE and TM. The recorded photo and the average distribution of the intensity of light (several horizontal lines of pixels) along the path of propagation are to be seen in Fig. 4.

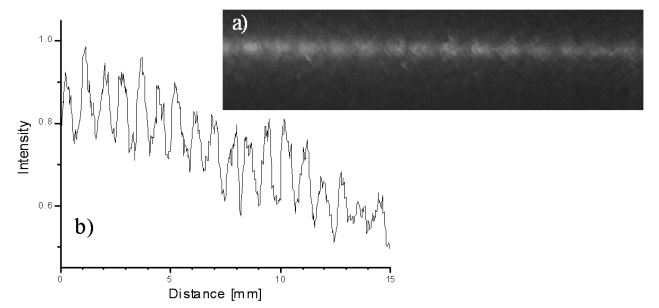

Fig. 4. (a) Image captured by the CCD camera (the polarizer was mounted in front of the camera). (b) Dependence of the intensity of scattered light. Analyzed images of excited modes $\left(\mathrm{TE}_{0}\right.$ and $\left.\mathrm{TM}_{0}\right)$.

Determining the distance between the first and the last distinct maximum (minimum) and the number of oscillations between them we determine the beat length and can calculate the modal birefringence of the investigated waveguide $\left(\lambda=635 \mathrm{~nm}\right.$ and $L_{\mathrm{B}}=0.84 \mathrm{~mm}$, the modal birefringence amounts to $\left.7.5 \times 10^{-4}\right)$.

\section{Conclusion}

The paper presents methods of measurements which permit to determine the attenuation and mode birefringence on only one test stand. In the case of semiconductor waveguides displaying considerable losses this method may prove to be troublesome. In waveguides with a high birefringence it may be difficult to excite simultaneously both orthogonal TE and TM modes. In many other planar waveguides, however, the presented methods of measurements make it possible to determine the attenuation and mode birefringence correctly in only one cycle of measurements.

Acknowledgments

The investigations were sponsored by the Ministry of Science and High Education of Poland within the grant O R00 017912.

\section{References}

[1] R.G. Hunsperger, Integrated Optics Theory and Technology, Springer, Berlin 2009.

[2] A. Boudrioua, J.C. Loulergue, Opt. Commun. 137, 37 (1997).

[3] R.K. Hickernell, D.R. Larson, R.J. Phelan Jr., L.E. Larson, Appl. Opt. 27, 2636 (1988).

[4] T. Feuchter, C. Thirstrup, IEEE Photon. Technol. Lett. 6, 1244 (1994).

[5] T.A. Strasser, M.C. Gupta, Appl. Opt. 31, 2041 (1992).

[6] F. Wang, F. Liu, G. Chang, Opt. Eng. 47, 024602 (2008).

[7] K. Gut, K. Nowak, Eur. Phys. J.-Spec. Top. 154, 89 (2008).

[8] P. Struk, T. Pustelny, K. Gut, K. Gołaszewska, E. Kaninska, M. Ekielski, I. Pasternak, E. Łusakowska, A. Piotrowska, Acta Phys. Pol. A 116, 414 (2009).

[9] K. Gut, D. Nabaglo, Acta Phys. Pol. A 116, 307 (2009).

[10] K. Gut, A. Zakrzewski, T. Pustelny, Acta Phys. Pol. A 118, 1140 (2010)

[11] K. Barczak, T. Pustelny, Z. Zycki, T. Blazejczak, Acta Phys. Pol. A 116, 250 (2009).

[12] K. Gut, J. Phys. IV JP (France) 129, 109 (2005).

[13] M. Szczurowski, W. Urbanczyk, M. Napiorkowski, P. Hlubina, U. Hollenbach, H. Sieber, J. Mohr, Appl. Opt. 50, 2594 (2011).

[14] T. Pustelny, J. Ignac-Nowicka, B. Jarząbek, A. Burian, Opt. Appl. 34, 551 (2004).

[15] K. Barczak, T. Pustelny, D. Dorosz, J. Dorosz, Europ. Phys. J. Spec. Top. 154, 11 (2008).

[16] K. Gut, Acta Phys. Pol. A 114, A121 (2008).

[17] A. Opilski, R. Rogoziński, K. Gut, M. Błahut, Z. Opilski, Opto-Electron. Rev. 8, 117 (2000).

[18] K. Gut, S. Drewniak, Acta Phys. Pol. A 118, 1134 (2010).

[19] K. Gut, T. Pustelny, D. Nabaglo, Acta Phys. Pol. A 118, 1136 (2010).

[20] K. Gut, P. Karasinski, W.T. Wójcik, R. Rogozinski, Z. Opilski, A. Opilski, Opt. Appl. 29, 101 (1999).

[21] K. Gut, S. Drewniak, Acta Phys. Pol. A 120, 630 (2011).

[22] C. Tyszkiewicz, T. Pustelny, Opt. Appl. 34, 507 (2004).

[23] K. Gut, Bull. Pol. Acad. Sci.: TS 59, 395 (2011).

[24] T. Pustelny, I. Zielonka, C. Tyszkiewicz, P. Karasinski, B. Pustelny, Opto-Electron. Rev. 14, 161 (2006).

[25] T. Pustelny, K. Barczak, K. Gut, J. Wojcik, Opt. Appl. 34, 531 (2004).

[26] T. Pustelny, M. Grabka, Acta Phys. Pol. A 116, 385 (2009).

[27] S.Y. Cheng, K.S. Chiang, IEEE Photon. Technol. Lett. 15, 700 (2003).

[28] W.W. Hu, K. Inagaki, Y. Mizuguchi, Opt. Lett. 26 , 193 (2001). 